\title{
ESTUDIO DE LA ANGIOGÉNESIS COMO FACTOR PRONÓSTICO DE LOS TUMORES VESICALES PT1G3
}

\author{
J.L. PALMERO MARTÍ, J.A. QUEIPO ZARAGOZA, J.L. RUIZ CERDÁ, \\ C.D. VERA DONOSO, L.A. RUBIO MARTÍNEZ*, F.J. VERA SEMPERE*, \\ J.F. JIMÉNEZ CRUZ
}

Servicio de Urología. *Servicio de Anatomía Patológica. Hospital Universitario La Fe. Valencia.

Actas Urol Esp. 28 (8): 594-601, 2004

\section{RESUMEN}

ESTUDIO DE LA ANGIOGÉNESIS COMO FACTOR PRONÓSTICO DE LOS TUMORES VESICALES pTIG3

INTRODUCCIÓN Y OBJETIVOS: La actividad angiogénica ha sido considerada como factor pronóstico en diversos tumores sólidos. Podemos analizarla de dos formas: determinación inmunohistoquímica de moléculas activadoras/inhibidoras de la angiogénesis y cuantificación de la densidad microvascular (DM). Nuestro objetivo es determinar en tumores vesicales pT1G3, el valor de la inmunohistoquímica del Vascular Endotelial Growth Factor (VEGF) y de la DM como factores pronóstico.

MATERIAL Y MÉTODOS: Estudio retrospectivo de 83 tumores vesicales pT1G3 con un seguimiento mínimo de 3 años. Analizamos la expresión del VEGF utilizando el Ac. monoclonal AbNo.360P. Para determinar la DM, marcamos los vasos (Ac.-FVIII) y cuantificamos el número de microvasos mediante un analizador digital de imagen, excluyendo aquellos que sobrepasan las 50 micras de diámetro. Correlacionamos los hallazgos con la recidiva, progresión y supervivencia, mediante tablas de contingencia (Chi-cuadrado) y curvas de Kaplan-Meier (Log-rank).

RESULTADOS: El tiempo medio de seguimiento ha sido de $58 \pm 28$ meses. El análisis Chi-cuadrado no muestra correlación ni con la recidiva ni con la supervivencia, pero si con la progresión: p(VEGF): 0,048, p(DM): 0,021 . Las curvas de Kaplan-Meier determinan diferencias significativas únicamente en el tiempo libre de progresión respecto de la DM (p:0,038).

CONCLUSIONES: La DM podría considerarse como factor pronóstico de progresión en base a estos resultados. No obstante son necesarios más estudios y un análisis multivariante que determine su aplicación clínica en este grupo de tumores de alto riesgo.

PALABRAS CLAVE: Angiogénesis. Densidad microvascular (DM). Factor crecimiento del endotelio vascular (VEGF).

\section{ABSTRACT}

STUDY OF THE ANGIOGENESIS AS PROGNOSTIC FACTOR OF pT1G3 BLADDER TUMOURS

INTRODUCTION AND OBJECTIVES: Angiogenic activity has been considered like prognostic factor in several solid tumors. This activity can be analysed by two ways: immunohistochemical determination of molecules that activate/inhibit angiogenesis or quantitive measure of microvascular density (MD). Our objective is to determine the prognostic value of Vascular Endothelial Growth Factor (VEGF) and Microvascular Density (MD) in pT1G3 bladder tumours.

MATERIAL AND METHODS: We have studied retrospectively 83 patients with pT1G3 tumors treated by TUR + endovesical instillations with a follow up of 3 years at least. We analysed VEGF expression monoclonal antibody № 360P. To determine MD we have marked vessels with FVIII antibody and detected "hot spots" areas. The number of microvessels is quantited by a digital image analyser excluding those that have more than 50 micras of diameter. We established the correlation of these findings with recurrence, progression and survival by using Chi-square test and Kaplan-Meier curves (log-rank).

RESULTS: Average follow up was $58+/$ - months. We have established like cut-off 50\% of tumor cells (VEGF) and 30 microvessels/fields (MD). Chi-square test did not show correlation with survival neither recurrence but it was positive for progression p(VEGF) 0.048 and p(DM) 0.021 . Kaplan Meier curves determined significative differences only for free of progression time respect to MD (p 0.038).

CONCLUSIONS: We did not find statistically significant value for recurrence nor survival. Just MD reached prognostic value for progression. More studies and multivariant analysis are required to determine the clinical utility of MD, specially in order to make more aggressive therapeutic options in this kind of patients.

KEY WORDS: Angiogenesis. Microvascular density (MD). Vascular endothelial growth factor (VEGF). 
$\mathrm{L}$ a angiogénesis se define como el crecimiento de nuevos vasos sanguíneos a partir de otros pre-existentes, inducido en el caso de las neoplasias, por las propias células tumorales. Ésta es un proceso necesario en el crecimiento tumoral, que permite no sólo el aporte de oxígeno y nutrientes, sino además el paso de células neoplasias al torrente circulatorio para su diseminación a distancia.

La existencia de actividad angiogénica en los tejidos tumorales se puede demostrar a través de la detección de moléculas angiogénicas o bien por el descubrimiento de la propia trama neovascular. Por ello, el estudio de la angiogénesis se puede abordar de dos formas: la cuantificación de la densidad microvascular (DM) y el análisis inmunohistoquímico de los niveles de los diferentes factores estimuladores/inhibidores de la angiogénesis.

En nuestro trabajo vamos a analizar ambas posibilidades; concretamente cuantificaremos la DM a partir del marcaje de los neovasos con el anticuerpo antifactor-VIII y determinaremos la expresión del factor angiogénico "Factor de Crecimiento del Endotelio Vascular" (VEGF).

Los problemas de hace unos años ligados a la evaluación de la angiogénesis limitaban su estudio como factor pronóstico; sin embargo las técnicas morfométricas e inmunohistoquímicas aplicadas más recientemente, constituyen una herramienta idónea para la valoración de ésta ${ }^{1,2}$.

\section{Factores angiogénicos}

En la angiogénesis existe un equilibrio dinámico entre los factores activadores e inhibidores, siendo tan importantes los efectos reguladores positivos de los primeros como los efectos negativos de los inhibidores ${ }^{3}$.

El VEGF o factor de permeabilidad vascular es una citoquina multifuncional que favorece la proliferación de las células endoteliales. Induce además la formación del factor estimulador de la uroquinasa plasminógena, lo que permite la transformación del plasminógeno en plasmita (factor de degradación de la matriz extracelular). También activa las colagenazas intersticiales, facilitando así la migración de las células endoteliales y neoplásicas. En estudios experimentales en los que se antagoniza el VEGF, se consigue inhibir el crecimiento, la angiogénesis y la invasión tumoral ${ }^{4}$.

De otra parte, son numerosos los grupos investigadores que han centrado su atención en la angiogénesis como factor pronóstico, valorando la DM en algunas patologías como el cáncer mamario ${ }^{5}$, cáncer colorectal $^{6}$, carcinoma gástri$\mathrm{co}^{7}$ y melanoma cutáneo ${ }^{8}$. Esto se ha visto favorecido por mejoras de metodología técnica que han acontecido en los últimos años, para efectuar una medición lo más exacta posible, con los denominados sistemas de análisis de imagen, que logran cuantificar la densidad de microvasos de una manera precisa y objetiva ${ }^{2,9}$. Esta metodología se basa en la tinción inmunohistoquímica de cortes desparafinados del tumor con anticuerpos que marcan específicamente las células endoteliales. Nosotros también hemos aplicado esta moderna metodología de análisis de imagen para determinar la densidad de microvasos en nuestra serie de tumores vesicales pT1G3.

\section{MATERIAL Y MÉTODOS}

Hemos realizado un estudio retrospectivo de una serie de 83 pacientes afectos de tumor vesical pT1G3, diagnosticados y tratados entre los años 1986 y 2003, en el Hospital Universitario La Fe de Valencia. Todos los pacientes cuentan con un seguimiento mínimo superior a tres años y fueron tratados inicialmente mediante resección transuretral completa (RTU), y una vez conocido su estadio y grado, los pacientes entraron en un protocolo de profilaxis endovesical con distintos agentes quimioterápicos.

En estos casos realizamos un estudio de la angiogénesis tumoral mediante la determinación de la DM y el estudio inmunohistoquímico del VEGF, tratando de establecer su validez como factor pronóstico.

\section{Estudio de la densidad microvascular}

Los microvasos tumorales fueron marcados utilizando el anticuerpo policlonal conocido como antígeno relacionado con el Factor VIII de la coagulación o factor de Von Willebrand (220M; Biomeda Foster City, CA, USA). De esta forma fue posible reconocer las zonas de máxima vascularización, también denominadas hot spots. Para el 
recuento de microvasos, se ha utilizado un analizador automático de imagen (MicroImage de Olympus) constituido por un ordenador 200 MMX, monitor y cámara digital $\mathrm{CCD}$, ésta última incorporada a un microscopio (Olympus $\mathrm{CH}-2$ ) (Fig. 1).

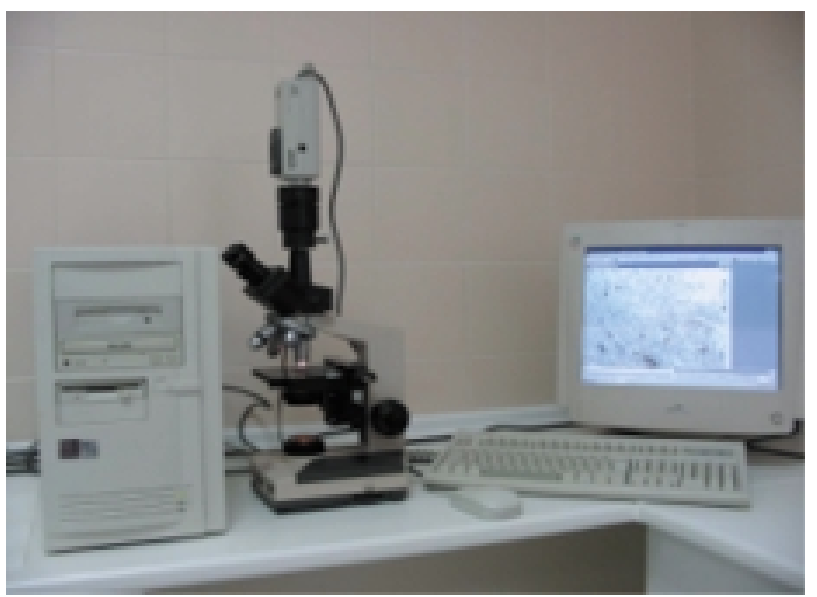

FIGURA 1. Analizador digital de imágenes.

Para la selección de la imagen correspondiente a la zona de máxima densidad microvascular o hot spots, se recorre la preparación a un aumento de 40x (4x de objetivo y 10x de ocular) hasta identificarla. Localizado el hot spot, se incrementa el número de aumentos hasta una magnificación total de 250x, con un campo real en el monitor de $0,5028 \mathrm{~mm}^{2}$. Mediante la cámara se capta la imagen del microscopio, que se traslada al ordenador y el monitor ofrece la imagen digitalizada. Esta imagen, formada por unidades denominadas pixels, se configura y guarda como una imagen color (opción RGB). A continuación, se realiza lo que se conoce como "segmentación de la imagen" en la que hay que seleccionar el color marrón de los vasos (por la reacción de la tetrahidrocloruro de diaminobencidina o DAB), frente al color azul-violeta del resto de las estructuras de la preparación teídas con hematoxilina. Una vez determinados los rangos de colores, se realiza el recuento automático de los microvasos, excluyendo los de mayor calibre (los que sobrepasan las 50 micras de diámetro), ya que éstos no se consideran como vasos neoformados ${ }^{10,11}$ (Figs. 2 y 3).

Los grupos (número de microvasos/0,5 mm2) que se establecieron fueron los siguientes: 1:0 -

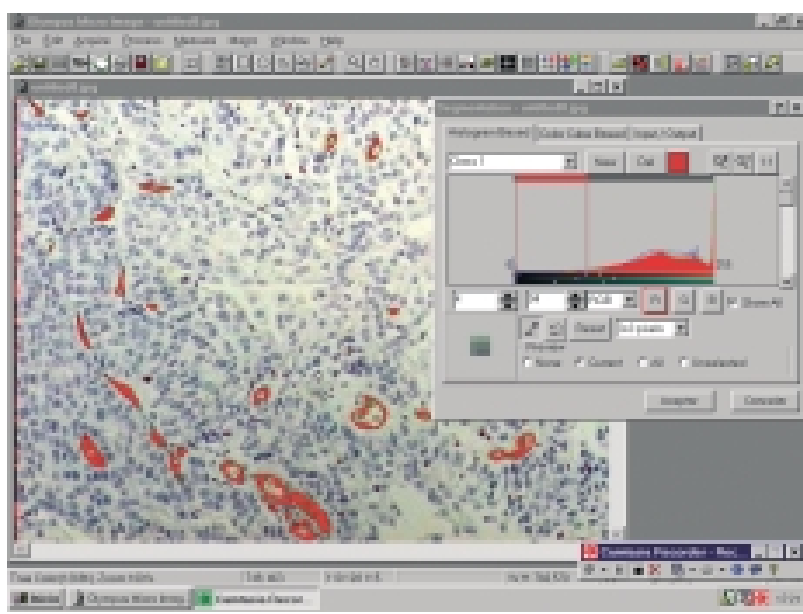

FIGURA 2. Proceso de segmentación de la imagen.

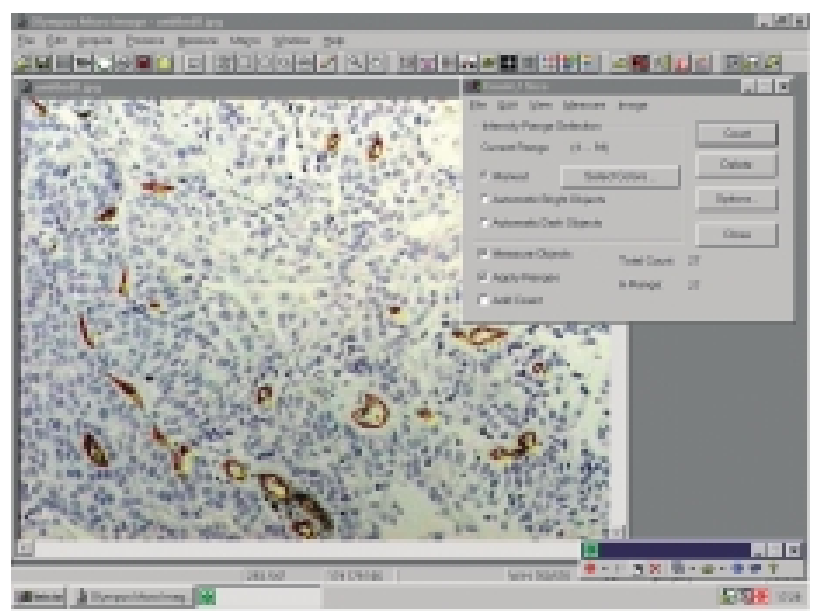

FIGURA 3. Microvasos marcados y seleccionados en el proceso de recuerdo $(250 x)$.

19, 2: 19 - 29, 3: $30-39,4: \geq 40$ microvasos; estableciendo el punto de corte en 30 , considerando entonces como alta o baja densidad microvascular los casos por encima o por debajo de dicho valor.

Con la utilización de este analizador de imágenes se consigue mayor precisión y fiabilidad en el recuento de vasos, eliminando el margen de subjetividad que acompaña a las mediciones realizadas por el observador a través del examen directo en el microscopio. Por tanto, su uso conlleva, como principal ventaja, una unificación de criterios en el estudio cuantitativo de la angiogénesis.

\section{Estudio del Factor de Crecimiento del Endotelio Vascular}

Para determinar la expresión del VEGF se utilizó el anticuerpo policlonal Abo.360P (BioGenex) 


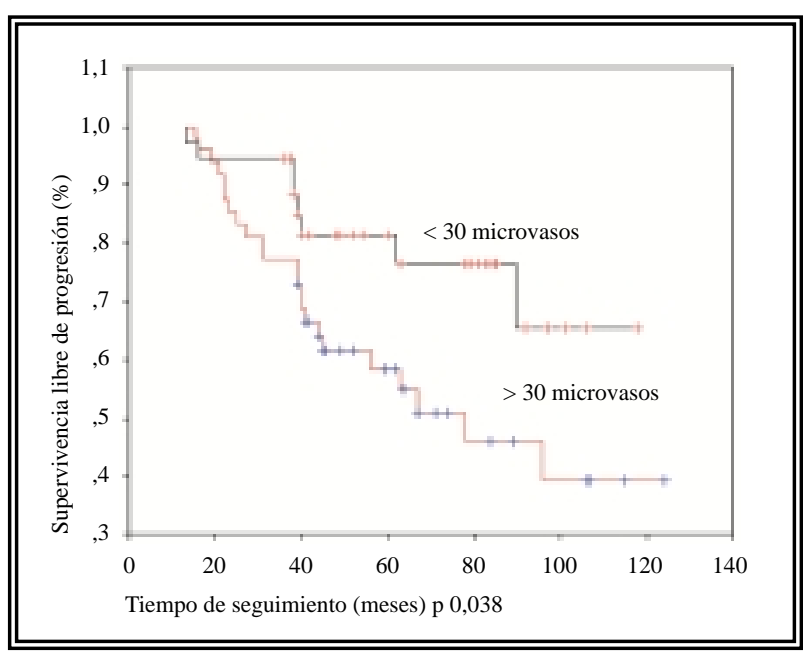

Gráfico 1a. Probabilidad de progresión en función de la densidad microvascular.

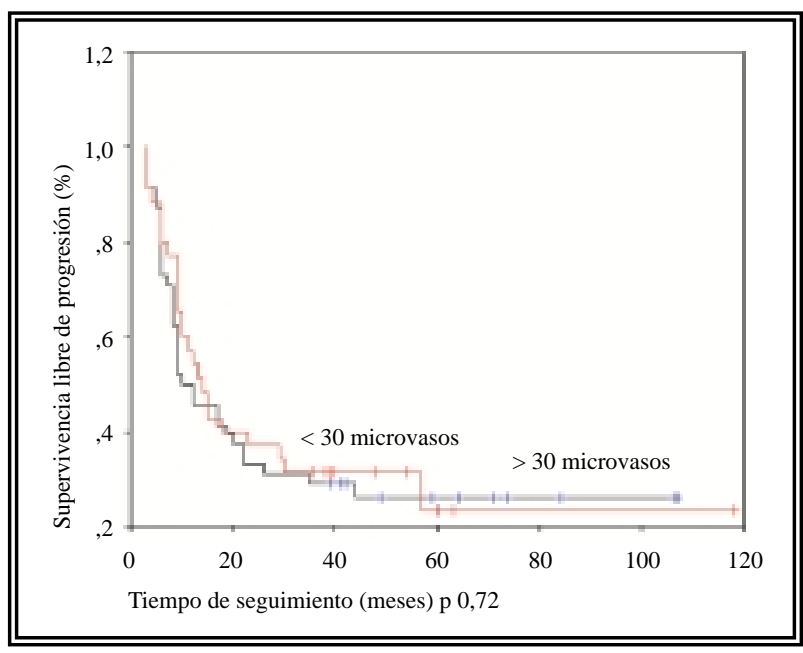

Gráfico $1 b$. Probabilidad de recidiva en función de la densidad microvascular.

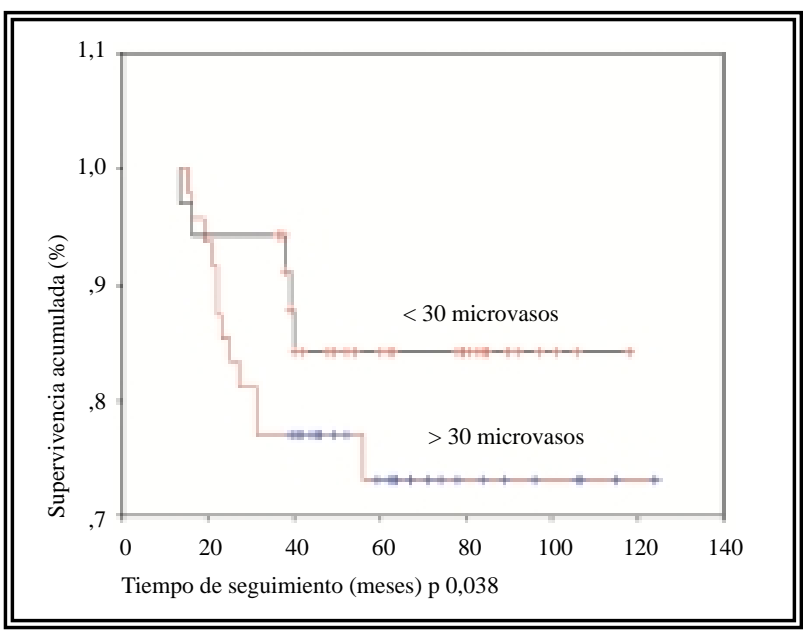

Gráfico 1c. Probabilidad de supervivencia en función de la densidad microvascular.

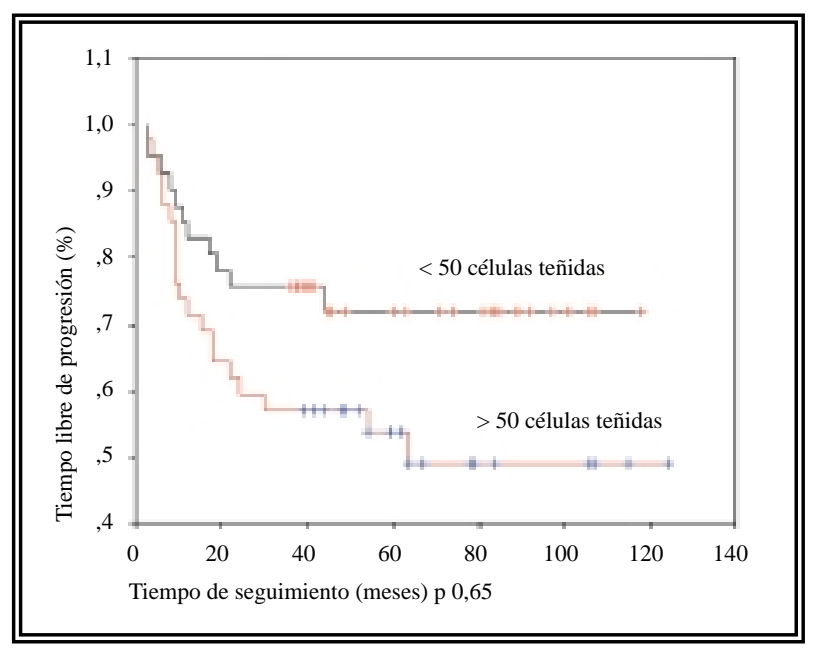

Gráfico 2a. Probabilidad de progresión en función del \% células teñidas (VEGF).

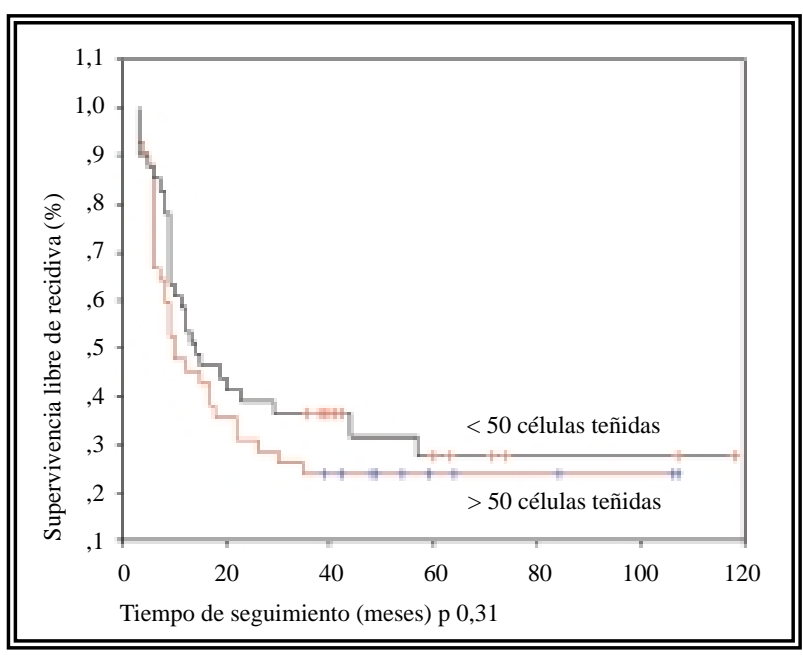

Gráfico $2 b$. Probabilidad de recidiva en función del \% células teñidas (VEGF).

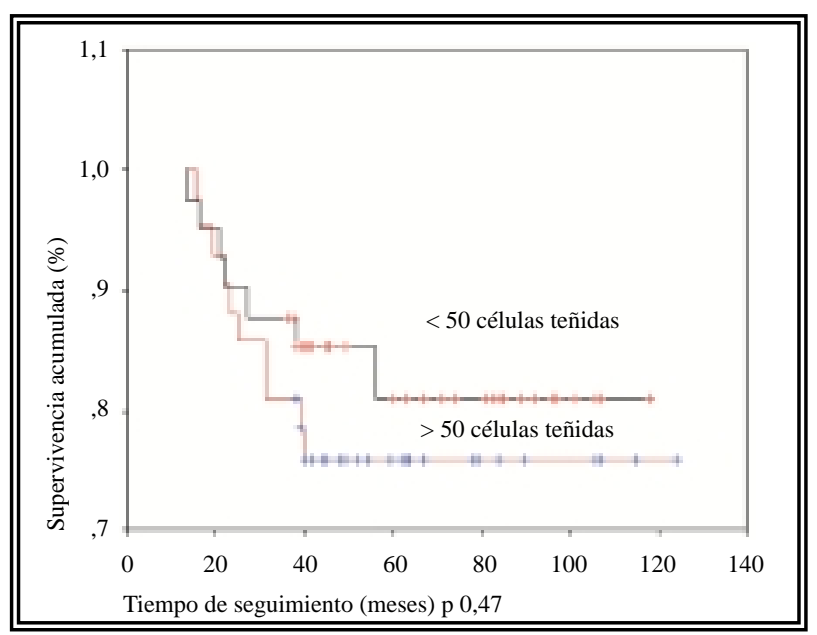

Gráfico 2c. Probabilidad de supervivencia en función del \% células teñidas (VEGF). 
Menarini Diagnostics), a una dilución 1:10. a partir del porcentaje de células marcadas se establecieron los siguientes grupos: 1: 0-24\%, 2: $25-49 \%$, 3: 50-74\%, 4: $\geq 75 \%$, estableciéndose para el análisis binario el punto de corte en el 50\%, considerando tinciones positivas las que superaban este punto de corte.

Estas determinaciones se llevaron a cabo sin conocimiento previo de la evolución clínica de los pacientes. Correlacionamos los hallazgos con la recidiva, progresión y supervivencia, mediante tablas de contingencia (Chi-cuadrado) y curvas de Kaplan-Meier (Log-rank).

\section{RESULTADOS}

De los 83 tumores, 57 (69\%) fueron primarios y $26(31 \%)$ procedían de un tumor previo de menor grado y/o estadio. El tiempo medio de seguimiento ha sido de $57,8 \pm 28,2$ meses (rango: 13-124 meses), mediana: 49 meses, presentando todos los casos un seguimiento mínimo de 36 meses (excepto los fallecidos por causa tumoral). Actualmente hay 49 pacientes vivos sin enfermedad, 4 vivos con enfermedad, 13 exitus por causa no tumoral y 17 exitus de causa tumoral.

Un total de 60 pacientes sufrieron recidiva (72\%). La mediana del tiempo libre de recidiva fue de 12 meses (I.C. 95\%: 7-17). En 20 pacientes la recidiva fue precoz (menor o igual 6 meses) y a los 12 meses ya había recidivado 42 de los casos. Sólo 2 pacientes tuvieron la primera recidiva a partir de los 3 años de seguimiento.

\section{Densidad microvascular}

La densidad microvascular media fue de 31,5 \pm 11,2 microvasos/campo y mediana: 32 . El punto de corte se estableció para 30 microvasos.

Las curvas de supervivencia pusieron de manifiesto que los pacientes con densidad microvascular alta presentaban un tiempo libre de progresión significativamente más corto que aquellos con baja densidad microvascular (p 0,038), no existiendo, sin embargo, diferencias estadísticamente significativas para el tiempo de libre de recidiva y de supervivencia (Gráficas 1a, 1b y 1c).

\section{Inmunotinción para VEGF}

El porcentaje medio de células teñidas fue del $51,9 \pm 24,2 \%$, mediana: $60 \%$. Establecimos el punto de corte en el 50\%, sin encontrar diferencias estadísticamente significativas en el tiempo libre de recidiva, tiempo de progresión y supervivencia, entre los pacientes asignados al grupo de "tinción positiva" y los asignados al de "tinción negativa" (Gráficas 2a, 2b y 2c).

De otra parte, al realizar el análisis Chi-cuadrado, para determinar la existencia de diferencias estadísticamente significativas, obtuvimos una ausencia de correlación con la recidiva y con la supervivencia. Sin embargo, con Chi-cuadrado obtuvimos correlación estadísticamente significativa para la progresión, tanto para la densidad microvascular como para VEGF: p (VEGF): 0,048, p (DM): 0,021.

\section{DISCUSIÓN}

\section{Valor pronóstico del VEGF}

El VEGF es el principal inductor de la neovascularización en el cáncer vesical y constituye una de las proteínas angiogénicas más estudiadas, no sólo desde un punto de vista pronóstico sino también en sus aplicaciones terapéuticas, y es junto a la determinación inmunohistoquímica de p53, Ki-67 y las metaloproteinasas de la matriz, uno de los marcadores pronósticos más prometedores en el cáncer vesical ${ }^{12}$. El aumento en la expresión del VEGF ha demostrado tener importancia pronóstica en muchos procesos neoplásicos, entre los que se encuentran el cáncer de mama y el de estómago; además se ha publicado que la inhibición de la expresión del VEGF usando anticuerpos específicos o induciendo receptores cuya zona de exposición queda oculta al VEGF provoca la inhibición del crecimiento tumoral y de la angiogénesis ${ }^{13-15}$.

La expresión del VEGF en el tejido vesical normal es significativamente menor que en el tejido tumoral, lo que ha conducido a la hipótesis de que la determinación del VEGF podría utilizarse como indicador pronóstico en el carcinoma vesical superficial. Aunque los niveles del VEGF condicionan la posterior formación de neovasos, su correlación con la densidad microvascular no es del todo evidente.

Respecto a la asociación con otras variables pronósticas clásicas como el grado y estadio, Chow NH y cols., sobre una serie de 185 casos de tumores superficiales (Ta, T1) encuentran corre- 
lación significativa con el grado tumoral ${ }^{16}$. Izawa JI y cols., aprecian un incremento significativo en la expresión del VEGF con el estadio, implicándolo en el desarrollo de la invasión muscular; además obtienen una expresión alta en el Cis, frente al tumor papilar, lo que pone de manifiesto su mayor expresión en tumores más agresi$\operatorname{vos}^{17}$. Otros autores, sin embargo, considerando conjuntamente tumores superficiales e invasivos, encuentran valores máximos de expresión del VEGF en los primeros ${ }^{18}$. Ello podría explicarse por el hecho de que en los tumores superficiales más agresivos, que van a progresar, los requerimientos de factores de crecimiento, incluido el VEGF, son mayores.

Algunos trabajos han considerado como factor pronóstico los niveles urinarios del VEGF, ya que ello constituye un marcador secundario de los niveles intratumorales, y por tanto podría utilizarse como indicador pronóstico indirecto ${ }^{19}$.

En el grupo específico de tumores superficiales, los trabajos recientes que consideran la expresión inmunohistoquímica del VEGF como factor predictivo de recurrencia, progresión o supervivencia son muy escasos; Chow $\mathrm{NH}$ y col., poseen la mayor serie, 186 tumores Ta T1; en su trabajo se muestran como factores pronósticos independientes el número de tumores y la infiltración de la lámina propia, no alcanzando valor pronóstico la inmunohistoquímica del $\mathrm{VEGF}^{16}$. Guo ZH y cols., obtienen, sin embargo, grandes diferencias en las tasas de recurrencia según la inmunohistoquímica del VEGF: los tumores con alta expresión del VEGF presentan una tasa de recurrencia del $61 \%$ frente a sólo el $16 \%$ en los tumores con baja inmunoreactividad ${ }^{20}$. Si consideramos el valor predictivo en tumores infiltrantes, el estudio de Inoue $\mathrm{K}$ y cols. muestra los niveles del VEGF como indicador del riesgo de metástasis, señalando que pacientes van a presentar mayor riesgo de metástasis, aunque se le realice un tratamiento radical con cistectomía o quimio/ radioterapia ${ }^{21}$.

A la vista de las publicaciones y resultados obtenidos no existe una suficiente información para sacar conclusiones a favor del uso clínico actual de este inmunomarcador. No hemos encontrado ningún trabajo que trate específicamente este factor en tumores T1G3.
Valor pronóstico de la densidad microvascular

Los vasos neoformados se caracterizan por presentar unas células endoteliales de carácter neoplásico, con un índice de proliferación aumentado, que puede llegar a ser de hasta 50 veces, células que configuran una red de microvasos que permite la invasividad y las metástasis ${ }^{22}$. Para la identificación y estudio de estos neovasos es necesario primero su tinción con anticuerpos específicos. En los diferentes trabajos publicados se han utilizado indistintamente como inmunomarcadores el anticuerpo anti-CD31, el anti-CD34 o el anti-Factor VIII, que se fijan específicamente a las células endoteliales, obteniéndose resultados similares con los distintos anticuerpos ${ }^{23}$. En nuestro estudio hemos utilizado el anti-Factor VIII o anti-Factor de Von Willebrand.

En la pasada década ya se puso de manifiesto que la densidad de microvasos era un factor pronóstico en el cáncer vesical infiltrante ${ }^{24,25}$, constituyendo el elevado número de vasos un factor predictivo de metástasis y muerte en tumores transicionales localmente avanzados ${ }^{26,27}$. Pero la reproductibilidad de su capacidad pronóstica en los tumores superficiales mínimamente invasivos (T1) sigue siendo actualmente objeto de controversia, debido fundamentalmente a que la estructura papilar de la mayoría de estos tumores dificulta la valoración de los microvasos.

Dinney CP y cols. han utilizado 2 modos de determinar la densidad microvascular: en el primero,o "estándar", se buscan las zonas de inmunotinción "calientes" o de mayor densidad de microvasos; en el segundo método, y para evitar el problema de la disposición papilar del núcleo fibrovascular, se realiza el contaje de los microvasos inmunoreactivos localizados entre la interfase del tumor y la lámina propia, o sea en la zona de invasión y probablemente de mayor actividad angiogénica. En su serie, el cálculo de las densidades mostró valores muy diferentes según el método utilizado, siendo mayores los recuentos netos de microvasos usando el método de la interfase tumor-lámina propia; pero no encontró correlación ni con la recidiva ni con la progresión tumoral, con ninguno de los dos métodos de análisis ${ }^{28}$.

Nosotros realizamos este estudio considerando que, puesto que la angiogénesis precede a la invasión, los tumores T1G3 destinados a invadir 
la capa muscular deberían tener incrementada la densidad de microvasos. Efectivamente hemos apreciado un incremento de actividad angiogénica en los tumores que posteriormente han progresado, reflejado en el análisis univariante (menor tiempo libre de progresión en aquellos tumores con más de 30 microvasos).

Algunos trabajos no analizan únicamente la densidad de microvasos sino que van más allá, determinando otras características morfométricas como el área vascular total, el perímetro vascular, etc. Korkolopoulou P y cols. analizaron, en una serie de 135 tumores vesicales, los siguientes parámetros morfométricos: densidad microvascular, perímetro vascular, área total, diámetros mayor y menor, compacticidad y forma de los microvasos. Utilizando el anticuerpo anti-CD31, obtuvieron en el análisis univariante, que en el caso de tumores superficiales el área vascular total y el diámetro vascular, constituían factores predictivos de supervivencia; y en el análisis multivariante, el área vascular total y la compacticidad de los neovasos, fueron los factores pronósticos independientes ${ }^{1}$.

Las publicaciones al respecto de la densidad microvascular como factor pronóstico en el cáncer vesical no son muy profusas, si las comparamos con los estudios inmunohistoquímicos que versan sobre las proteínas del ciclo celular; la mayoría de los trabajos no consideran la densidad microvascular como factor pronóstico clínicamente útil en tumores superficiales, siendo en los tumores infiltrantes donde con más frecuencia si presenta valor pronóstico independiente2 1,23-25,27,29-33 (Tabla 1).

En relación al objetivo de nuestro trabajo, Dinney CP y cols. analizaron la capacidad predictiva de la densidad microvascular en relación a la identificación de los pacientes en estadio $\mathrm{T} 1$ que deberían someterse a una cistectomía temprana; sobre una serie de 54 pacientes T1 la densidad microvascular no predijo ni recidiva ni la progresión, considerando por tanto que no constituye un marcador pronóstico y no tiene validez para seleccionar a estos pacientes $^{28}$. Sin embargo una serie más amplia y reciente, de Goddard JC y cols. sobre un total de 170 tumores superficiales encuentran que la
Tabla 1

Principales trabajos sobre el valor pronóstico de la densidad microvascular

\begin{tabular}{|c|c|c|c|}
\hline Estudio & $\begin{array}{c}\text { № } \\
\text { tumores }\end{array}$ & Estadio & $\begin{array}{l}\text { Valor pronóstico } \\
\text { independiente }\end{array}$ \\
\hline Goddard JC (2003) & 170 & Ta, T1 & Si (progresión) \\
\hline $\begin{array}{l}\text { Elsobky E } \\
(2002)\end{array}$ & 154 & $\begin{array}{l}\text { Tumores } \\
\text { superf/ } \\
\text { infiltrantes }\end{array}$ & Si (supervivencia) \\
\hline $\begin{array}{l}\text { Offersen BV } \\
(2002)\end{array}$ & 107 & $\begin{array}{l}\text { Tumores } \\
\text { superf/ } \\
\text { infiltrantes }\end{array}$ & No \\
\hline Reiher F (2002) & 84 & Ta, T1 & No \\
\hline $\begin{array}{l}\text { Korkoloupoulou P } \\
(2001)\end{array}$ & 135 & $\begin{array}{l}\text { Tumores } \\
\text { superf/ } \\
\text { infiltrantes }\end{array}$ & $\begin{array}{l}\mathrm{Si} \\
\text { (área/ } \\
\text { compacticidad) }\end{array}$ \\
\hline $\begin{array}{l}\text { Inoue } \mathrm{K} \\
(2000)\end{array}$ & 55 & $\begin{array}{l}\text { Tumores } \\
\text { infiltrantes }\end{array}$ & No \\
\hline $\begin{array}{l}\text { Chaudhary R } \\
\text { (1999) }\end{array}$ & 88 & $\begin{array}{l}\text { Tumores } \\
\text { superf/ } \\
\text { infiltrantes }\end{array}$ & $\mathrm{Si}$ \\
\hline Ozer E (1999) & 20 & T1G3 & $\mathrm{Si}$ \\
\hline Dinney CP (1998) & 88 & $\mathrm{~T} 1$ & No \\
\hline
\end{tabular}

densidad microvascular es un factor pronóstico independiente de progresión a un estadio infiltrante y por tanto puede seleccionar los pacientes para cistectomía ${ }^{29}$.

Si nos centramos específicamente en tumores T1G3, únicamente hemos encontrado un trabajo reciente que analice la densidad microvascular34, sobre una serie de sólo 20 pacientes, marcando microvasos con el anti-factor VIII, obtiene valor pronóstico significativo tanto de recurrencia como de progresión, pero el propio autor advierte que se trata de una serie pequeña y de un seguimiento corto.

\section{CONCLUSIONES}

En base a los resultados de nuestro trabajo, la densidad microvascular podría considerarse como un factor pronóstico de progresión en el tumor pT1G3. Sin embargo, el incremento de la angiogénesis, estimado por el recuento de microvasos, no presenta actualmente datos suficientes que demuestren su valor como marcador útil en la selección de los pacientes con tumor vesical T1G3, que puedan ser candidatos adecuados para cistectomía. 


\section{REFERENCIAS}

1. Korkolopoulou P, Konstantinidou AE, Kavantzas N et al. Morphometric microvascular characteristics predict prognosis in superficial and invasive bladder cancer. Virchows Arch 2001;438:603-611.

2. Qian CN, Min HQ, Liang XM et al. Primary study of neovasculature correlating with metastatic nasopharyngeal carcinoma using computer image analysis. J Cancer Res Clin Oncol 1997;123:645-651.

3. Campbell SC, Volpert OV, Ivanovich M et al. Molecular mediators of angiogenesis in bladder cancer. Cancer Res 1998;58:1298-1304.

4. Skobe M, Rockwell P, Goldstein N et al. Halting angiogenesis suppresses carcinoma cell invasion. Nat Med 1997;3:1127-1222.

5. Tas F, Yavuz E, Aydiner A et al. Angiogenesis and p53 protein expresión in breast cancer: prognostic roles and interrelationships. Am J Clin Oncol 2000;23:546-553.

6. Engel CJ, Bennett ST, Chambers AF et al. Tumor angiogenesis predicts recurrence in invasive colorectal cancer when controlled for Dukes staging. Am J Surg Pathol 1996;20: 1260-1265.

7. Sanz-Ortega J, Steinberg SM, Moro E et al. Comparative study of tumor angiogenesis and immunohistochemistry for p53, c-ErbB2, c-myc and EGFr as prognostic factors in gastric cancer. Histol Histopathol 2000;15:455-462.

8. Kashani-Sabet M, Sagebiel RW, Ferreira CM et al. Tumor vascularity in the prognostic assessment of primary cutaneous melanoma. J Clin Oncol 2002;20:1826-1831.

9. Rubio L, Burgos JS, Morera C et al. Morphometric study for tumor angiogenesis as a new prognostic factor in nasopharyngeal carcinoma patients. Pathol Oncol Res 2000;6:210-216.

10. Tanigawa N, Matsumura M, Amaya $\mathrm{H}$ et al. Tumor vascularity correlates with the prognosis of patients with esophageal squamous cell carcinoma. Cancer 1997;79: 220-225.

11. Weidner N, Semple JP, Welch WR et al. Tumor angiogenesis and metastasis-correlation in invasive breast carcinoma. N Engl J Med 1991;324:1-8.

12. Kausch I, Bohle A. Molecular aspects of bladder cancer III. Prognostic markers of bladder cancer. Eur Urol 2002;41: 15-29.

13. Maeda K, Chung YS, Ogawa Y et al. Prognostic value of vascular endothelial growth factor expresión in gastric carcinoma. Cancer 1996;77:858-863.

14. Millauer B, Longhi MP, Plate KH et al. Dominant-negative inhibition of Flk-1 suppresses the growth of many tumor types in vivo. Cancer Res 1996;56:1615-1620.

15. Warren RS, Yuan H, Matli MR et al. Regulation by vascular endothelial growth factor of human colon cancer tumorigenesis in a mouse model of experimental liver metastasis. J Clin Invest 1995;95:1789-1797.

16. Chow NH, Liu HS, Chan SH et al. Expression of vascular endothelial growth factor in primary superficial bladder cancer. Anticancer Res 1999; 19: 4593-4597.

17. Izawa JI, Slaton JW, Kedar D et al. Differential expression of progression-related genes in the evolution of superficial to invasive transitional cell carcinoma of the bladder. Oncol Rep 2001;8:9-15.

18. O’Brien T, Cranston D, Fuggle S et al. Different antiogenic pathways characterize superficial and invasive bladder cancer. Cancer Res 1995;55:510-513.
19. Jones A, Crew J et al. Vascular endothelial growth factor and its correlation with superficial bladder cancer recurrence rates and stage progression. Urol Clin North Am 2000;27:191-197.

20. Guo ZH, Mei H, Huang $J$ et al. Vascular Endothelial Growth Factor (VEGF) and Basic Fibroblast Growth Factor (bFGF) expression in superficial transitional cell bladder carcinoma. Ai Zheng (Abstract) 2003;22:307-309.

21. Inoue $\mathrm{K}$, Slaton JW, Karashima $\mathrm{T}$ et al. The prognostic value of angiogenesis factor expression for predicting recurrence and metastasis of bladder cancer after neoadjuvant chemotherapy and radical cystectomy. Clin Cancer Res 2000;6:4866-4873.

22. Harris AL. Antiangiogenesis for cancer therapy. Lancet 1997;349:13-15.

23. Reiher F, Ozer O, Pins M et al. p53 and microvessel density in primary resection specimens of superficial bladder cancer. J Urol 2002;167:1469-1474.

24. Bochner BH, Cote R, Groshen S et al. Angiogenesis in bladder cancer: relationship between microvessel density and tumor prognosis. J Natl Cancer Inst 1995;87:16031612.

25. Dickinson AJ, Fox S, Persad RA et al. Quantification of angiogenesis as an independent predictor of prognosis in invasive bladder carcinomas. Br J Urol 1994;74:762-766.

26. Crew JP, O'Brien TS, Harris AL et al. Bladder cancer angiogenesis, its role in recurrence, stage progression and as a therapeutic target. Cancer Metastasis Rev 1996;15:221-230.

27. Jaeger $\mathrm{T}$, Weidner $\mathrm{N}$, Chew $\mathrm{K}$ et al. Tumour angiogenesis correlates with lymph node metastases in invasive bladder cancer. J Urol 1995;154:69-71.

28. Dinney CP, Barkowski RC, Antelo M et al. Relationship among cystectomy, microvessel density and prognosis in stage T1 transitional cell carcinoma of the bladder. J Urol 1998;160:1285-1290.

29. Goddard JC, Sutton CD, Furness PN et al. Microvessel density at presentation predicts subsequent muscle invasion in superficial bladder cancer. Clin Cancer Res 2003;9:2583-2586.

30. Offersen BV, Knap MM, Marcussen N et al. Intense inflammation in bladder carcinoma is associated with angiogenesis and indicates good prognosis. Br J Cancer 2002;87: 1422-1430.

31. Chaudhary R, Bromley M, Clarke NW et al. Prognostic relevance of micro-vessel density in cancer of the urinary bladder. Anticancer Res 1999;19:3479-3484.

32. Hawke CK, Delahunt B, Davidson PJ. Microvessel density as a prognostic marker for transitional cell carcinoma of the bladder. Br J Urol 1998;81:585-590.

33. Philip EA, Stephenson TJ, Reed MW. Prognostic significance of angiogenesis in transitional cell carcinoma of the human urinary bladder. Br J Urol 1996;77:352-357.

34. Ozer E, Mungan MU, Tuna B et al. Prognostic significance of angiogenesis and immunoreactivity of cathepsin D and type IV collagen in high-grade stage T1 primary bladder cancer. Urology 1999;54:50-55.

Dr. J.L. Palmero Martí

Servicio de Urología. Hospital Univ. La Fe

Avda. Campanar, 21

46009 Valencia

(Trabajo recibido el 29 marzo de 2004) 